\author{
Wiesław Mateusz Malinowski \\ avec la collaboration d'Alicja Sobierajska \\ Adam Mickiewicz University \\ ul. Wieniawskiego 1 , \\ 61-712 Poznań, Poland
}

\title{
Sur la conjonction de l'ophélique et du narcissique dans l'œuvre poétique de Georges Rodenbach
}

\begin{abstract}
The paper examines a special place that the characters of Ophelia and Narcissus, so closely connected with the motif of water, occupy in the poetic imaginarium of George Rodenbach. What we deal with here is an unhealthy obsession within which two characters of literature and mythology, unhappily in love, are presented as two alter egos of the poet himself. An endless sequence of images associating water with death reveals the fascination and trepidation of the symbolist, who uses the Opheliac depths and Narcissus's water mirror as a reflection of different states of his soul.

Keywords: Rodenbach; symbolism in poetry; Ophelia; Narcissus
\end{abstract}

Dès 1886, l'eau parle sous la plume de Rodenbach, s'adressant aux amoureux dans un poème de La Jeunesse blanche :

Elle a des paroles mouillées

Et chuchote, avec des serments,

Qu'ils s'en iront sous les feuillées.

Qu'ils s'en iront au bon soleil 
Chercher au fond de la campagne

Un pacifique, un long sommeil

Qu'un rêve de fleurs accompagne !...

Cette voix ne laisse pas le jeune poète indifférent, puisqu'il proclame aussitôt :

Et parfois des poètes doux

Que la voix de l'eau triste appelle,

Escaladent les garde-fous

Pour aller dormir avec elle.

Puisque personne n'a compris

Combien leur Ame est grande et fière,

Ils offriront leurs yeux flétris

Aux baisers froids de la Rivière !

(Rodenbach 1886, L'eau qui parle : 111-112)

Cette attirance morbide de l'eau ne fera qu'augmenter avec le temps dans son œuvre où, tout naturellement, elle prendra pour protagonistes privilégiés deux figures hydriques, deux amoureux célèbres et malheureux empruntés à l'histoire littéraire ou à la mythologie, présentés plus ou moins ouvertement, disons-le tout de suite, comme deux alter ego du poète.

Dans Le Règne du silence, recueil de 1891, le poète évoque déjà les secrètes relations que son âme entretient avec Bruges en tant que ville d'eau, dressant un décor urbain où, parmi les «glaives des roseaux » et «les eaux prisonnières » plane l'ombre de la figure sororale d'une ville anthropomorphisée, d'une ville ophélisée :

Ô ville, toi ma sœur à qui je suis pareil [...],

Nous sommes tous les deux la ville en deuil qui dort [...] ;

Plus de bruits, de reflets... Les glaives des roseaux

Ont un air de tenir prisonnières les eaux,

Les eaux vides, les eaux veuves, où le vent seul

Circule comme pour les étendre en linceul... 
N'est-ce pas déjà le fil conducteur du futur roman? Dans Bruges-laMorte, le nom de la noyée shakespearienne apparaîtra explicitement, et à deux reprises, au point de permettre à Gaston Bachelard d'interpréter le roman comme "l'ophélisation d'une ville entière » (Bachelard 1942 : 121), même si la femme du protagoniste n'est pas morte noyée. Une voix insinuante y monte en effet du fond du canal et invite l'homme à se laisser engloutir. La formule bachelardienne a été maintes fois répétée et le roman analysé sous cet angle (p. ex. Malinowski 2005). Cependant, la figure d'Ophélie flotte plus constamment encore sur les eaux dans l'univers poétique de Rodenbach et son image ne cesse d'y interpeller l'auteur. C'est aux modalités de cette présence, explicite ou latente, que nous allons nous intéresser ici.

Dans sa thèse de doctorat soutenue en 2014 à l'Université Adam Mickiewicz de Poznań, Alicja Sobierajska a bien montré le caractère composite du mythe d'Ophélie, «amalgame de différentes aspirations philosophiques, psychologiques ou artistiques des auteurs », en l'occurrence des écrivains français et belges de la deuxième moitié du $\mathrm{XIX}^{\mathrm{e}}$ siècle (Sobierajska 2014 : 49). La jeune noyée shakespearienne y apparaît en effet comme un être aux visages multiples, empruntés à des figures diverses qui dominent le panthéon littéraire de l'époque symboliste. En tout premier lieu, à celle de Narcisse, telle que nous la livre l'invariant ovidien, soit le Narcisse qui finit son aventure dramatique par ne jamais consommer son amour, tout comme Ophélie.

Narcisse et Ophélie, homme et femme, l'un scrutant la surface de l'eau, l'autre pénétrant dans sa profondeur, figures si différentes à certains égards, comme le montre Julien Eymard (Eymard 1977 : 145 et passim), demeurent pourtant semblables à bien d'autres. Leurs similitudes ne se limitent nullement à des facteurs tels que la beauté insolite du jeune corps, la solitude amoureuse, les apparences de la folie, la fin tragique de la vie au bord de l'eau ou dans ses profondeurs. Leurs mythes se croisent dans des aspects autrement essentiels. En méprisant sa jeunesse et sa beauté, Ophélie attente à ses jours et rentre à la matrice de la terre, se fond avec la nature, se 
dissout en quelque sorte dans l'eau. Narcisse, lui, se transforme en une fleur belle et odorante auprès de la source ; selon certaines versions du mythe, il se serait même noyé en voulant embrasser sa propre image reflétée par l'eau et des fleurs blanches seraient apparues à l'endroit de sa disparition. Dans les deux mythes, par leur contact intime avec l'eau, les héros se détournent du corporel pour s'ouvrir à une réalité autre.

Et c'est précisément cela qui intéresse les écrivains du symbolisme, dont Georges Rodenbach. L'image du canal de Bruges qui apparaît dans Le Règne du silence correspond manifestement chez le poète à sa tentative de dévoiler les mystères de son propre être.

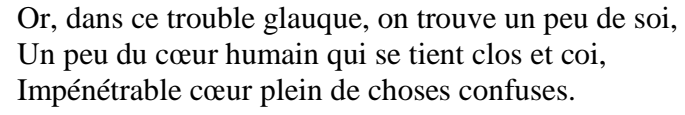

(Rodenbach 1891, Le Ceur de l'eau XV: 69)

C'est que, à la dimension mortuaire de l'eau s'ajoute immédiatement sa fonction spéculaire. Tout au long du recueil de 1891, et cela d'une manière quasi obsessionnelle, c'est l'âme du poète qui, à force de se refléter dans l'eau, se fait elle-même aquatique, contaminant à l'occasion tout ce qui l'entoure, multipliant les surfaces réfléchissantes. Dans le premier cycle du Règne du silence, intitulé $L a$ Vie des chambres, se développe longuement en effet une métaphore filée où c'est le mobilier des chambres habitées par le poète qui se liquéfie, à l'image de son âme. À commencer par le miroir, qui offre au poète la possibilité d'un jeu de mots subtil avec la glace :

...Mon rêve dort

Dans le profond miroir, comme en un canal mort ;

Et faut-il désirer un coup de vent qui chasse

En pleine mer, cette âme à l'ancre dans la glace ?

(Rodenbach 1891, La Vie des chambres III : 8) 
Miroir qui reprendra trois pages plus loin cette dimension quelque peu narcissique :

\footnotetext{
Le miroir est l'amour, l'âme-sœur de la chambre

Où tout d'elle : le lustre en fleur, les bahuts vieux,

La statuette au dos de bronze qui se cambre,

Se réfléchit en un hymen silencieux.

Car l'amour n'est-ce pas n'être plus seul et n'est-ce

Pas se doubler par un autre meilleur que soi ?
}

(Rodenbach 1891, La Vie des chambres $V: 11$ )

L'image du lustre en verre de Venise, comparé par le poète à « une fontaine blanche aux givres équivoques, jet d'eau gelé, mais où l'eau souffre encor ... », lui offre elle aussi sa fonction de miroir :

Ce lustre, c'est mon Cour visible en ce décor

Qui frissonne en sourdine et sans cesse s'afflige,

Jet d'eau fleurdelisé dont la plainte se fige !

(Rodenbach 1891, La Vie des chambres X : 21-22)

La présence du piano, dans le sixième poème de La Vie des chambres, suscite pour sa part le rêve d'une musique qui, conjuguée avec les motifs de l'eau, des fleurs et de la lune, semble nourrir l'idée de la mort ophélique, de la mort à apprivoiser :

Quelle ivresse si, par un soir doux de printemps,

Quelque vierge attirée à sa mélancolie

Ressuscitait de lui tous les rythmes latents :

Gerbe de lis blessés que son jeu lent délie ;

Eau pâle du clavier où son geste amusé

- Rafraîchi comme ayant joué dans une eau claire -

Ferait surgir un blanc cortège apprivoisé...

(Rodenbach 1891, La Vie des chambres VI : 13-14)

Derrière cette musique qui, dans la chambre à la fenêtre ouverte, déferle tristement et «à petites vagues » en faisant à l'âme du poète « un mal presque physique », mélodie «confuse comme un songe », 
dont on ne sait pas finalement si elle vient d'un piano, d'un violon ou d' " une voix humaine en élans comme une eau », et qui "sinue en forme de ruisseaux », il est certes permis d'entendre l'écho de la chanson d'Ophélie. En entendant ses lignes du son, le poète croit pouvoir «suivre et lire un peu » sa propre destinée (Rodenbach 1891, La Vie des chambres XIV: 30-31). Il en est encore ainsi des vitres sous la pluie dans une obscurité qui inquiète le poète, qui a « de félines étreintes comme une eau de canaux traitres où l'on se noie » (Rodenbach 1891, La Vie des chambres VIII : 17), vitres qui font surgir dans sa rêverie un «visage exsangue couronné par des épines d'eau... » (Rodenbach 1891, La Vie des chambres XVI : 35).

Ayant inspecté ainsi ses chambres silencieuses, avec tout leur potentiel aquatique, ophélique et narcissique à la fois, l'auteur du Règne du silence décide de scruter, avec ses yeux angoissés, Le Cour de l'eau. Plongeant son regard dans le bassin d'eau dormante, «eau nostalgique et qu'un regret tourmente », le poète découvre ce qu'il appelle l'œil de l'eau, « œil bénin, œil de femme où tout un ciel se rêve »; le voilà en proie à un rêve étrange où Narcisse semble vouloir rejoindre Ophélie :

- Oh ! L'émoi de descendre en cet iris profond

Et dans cette prunelle où les nuages vont !

Mais « l'ivresse de s'y rêver divin est brève », constate le poète,

Car on se heurte vite aux si courtes parois,

Quand le cristal se brise en brusques désarrois

Et qu'un gouffre mortel, quoique exigu, succède

À tout cet infini qu'on supposait dans l'eau !

(Rodenbach 1891, Le Cæur de l'eau V:50)

Le rêve que suscite l'eau tentatrice semble désormais céder place à la désillusion, voire au désespoir.

Mensonge équivalent d'un œil cher, d'un œil beau

Qu'on voudrait habiter comme une source tiède

Où l'azur sans limite irait à l'infini. 
Mais le voyage aussi dans cet œil n'est qu'un leurre,

Car derrière l'iris au cristal aplani

L'amour naï, qui plonge au fond, soudain s'épeure,

Se heurte et se fait mal à la froideur du cœur,

Dont le néant si proche est une vasque étroite.

(Rodenbach 1891, Le Cour de l'eau V: 51)

La descente au fond de l'eau donnera lieu à une véritable vivisection qu'opère le poète sur sa propre âme, âme dans laquelle défilent les souvenirs d'êtres disparus, mais « sans cesse émergeants », où ses propres détresses se reflètent dans les détresses d'Ophélie et l'amènent à réfléchir sur son destin.

Aux vitres de notre âme apparaissent le soir

Des visages anciens demeurés dans le verre $[\ldots]$;

Faces mortes toujours près de s'évanouir

Et sans cesse émergeant, - sitôt qu'on les oublie, -

Au fil de l'âme, en des détresses d'Ophélie

Dont les cheveux de lin ont un air de rouir...

\section{Et le poète de s'exclamer :}

Ah! comment essayer d'avoir un peu de joie Quand les vitres de l'âme aimante sont de l'eau

Où reparaît sans cesse et sans cesse se noie

Un doux visage intermittent dans un halo !

(Rodenbach 1891, Au fil de l'âme IX : 166-167)

Véhiculée par l'image de l'eau ophélique et celle des cheveux éparpillés de la noyée, la question ne cesse de se poser sous la plume de Rodenbach. Mais ce n'est pas pour rien que l'EAU s'écrit dans tout le recueil avec la majuscule; elle devient malgré tout pour le poète une «sœur de charité pour qui souffre » et « que n'a pu satisfaire aucune joie humaine », l'incarnation d'une séductrice sui generis qui attire ses contemplateurs dans d'obscures profondeurs, en sorte que peu d'entre eux peuvent résister à sa tentation :

Son amour du repos, son dégoût de la vie 
Sont si contagieux que plus d'un l'a suivie

Dans la chapelle d'ombre, au fond pieux des eaux,

Où, tranquille, elle chante au pied des longs roseaux...

(Rodenbach 1891, Le Cour de l'eau XI : 62)

L'eau de Rodenbach, «pensive et nostalgique », possède « le cœur de vierge exaltée en proie à l'idéal », qui « souffre d'être seule » parmi « les grands saules lunaires» (Rodenbach 1891, Le cœur de l'eau VI : 54, 52). N'est-ce pas le cœur d'Ophélie ? Ayant cherché en vain le courage de vivre, les rêveurs de l'eau, tous ceux dont les âmes, comme celle du poète, comme celle de la fiancée d'Hamlet, « ont trop souffert aux chemins du Réel » (Rodenbach 1891, Au fil de l'âme IV: 155), se laissent facilement envoûter par son chant lénifiant et fluide. Finalement, ils cèdent à ses influences moribondes :

Elle chante ! Elle dit : « Les doux abris que j’ai

Pour ceux de qui le cœur est trop découragé... »

$\mathrm{Ah}$ ! La molle attirance et quelle voix divine !

Car, pour leur fièvre, c'est la fraîcheur d'un bon lit !

Et beaucoup, aimantés par cet appel propice,

Perclus, entrent dans l'Eau comme on entre à l'hospice,

Puis meurent. L'Eau les lave et les ensevelit

Dans ses courants aussi frais que de fines toiles ;

Et c'est enfin vraiment pour eux la Bonne Mort.

(Rodenbach 1891, Le Cour de l'Eau XI : 62-63)

Largement présent dans Le Règne du silence, l'imaginaire ophélique et narcissique revient en force dans Les Vies encloses, recueil de 1896. L'eau d'un aquarium, cette fois-ci, où le regard « descend et plonge » dans un poème au titre hautement significatif d'Aquarium mental, se laisse voir non plus en horizon, reflétant «le fard délayé du visage des Ophélies » (Rodenbach 1896, Aquarium mental II : 6), mais « dans sa profondeur, son infini de songe, sa vie intérieure, à nu sous la cloison », « tout autre qu'à la surface » (p. 5). Nous voyons le corps d'Ophélie descendre au fond de l'eau dans une 
scène de noyade qui n'est pas sans rappeler le tableau de Paul Albert Steck, présenté au Salon de 1895, soit quelques mois avant la parution du recueil de Rodenbach, une des rares toiles à représenter Ophélie dans la pose verticale, descendante.

Ophélie a laissé sombrer à pic ses nattes

Qui se sont peu à peu tout à fait dénouées ;

Ses yeux ouverts sur l'eau sont comme deux stigmates ;

Ses mains pâles sont si tristement échouées ;

Pourtant elle sourit, sentant sur son épaule

Ruisseler tout à coup sa chevelure immense,

Qui la fait ressembler au mirage d'un saule.

« Suis-je ou ne suis-je pas »? a songé sa démence...

Les cheveux d'Ophélie envahissent l'eau grise,

Tumulte inextricable où sa tête s'est prise.

(Rodenbach 1896, Aquarium mental III : 8)

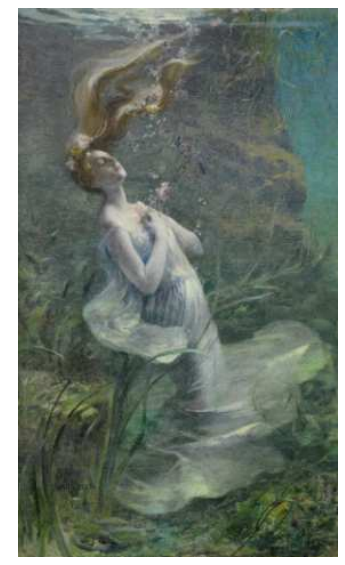

Paul Steck, Ophélie, 1895

Le pinceau du peintre saisit Ophélie au moment où elle cesse de flotter à fleur de l'eau et, à la suite de l'action des forces de gravité, commence à immerger dans les profondeurs aquatiques dont le fond rocheux se présente comme résolument hostile, menaçant et sinistre. L'héroïne descend dans ce monde sous-marin en prenant la pose d'une personne en prière, ou dans une extase inexplicable. Ses mains, à l'instar du défunt reposant dans le cercueil, sont croisées sur la poitrine ; yeux mi-clos, visage rengorgé vers la surface qui s'éloigne, cheveux librement éparpillés, marquant son dernier chemin par le sillage qui se peint derrière elle, Ophélie se laisse engloutir dans cette position verticale.

Sous la plume de Rodenbach, l'image d'Ophélie commence à s'estomper au moment où les cheveux de la noyée, ruisselant sur son épaule, la faisant « ressembler au mirage d'un saule ", « envahissent l'eau grise ». C'est alors que se pose dans son esprit la 
question identitaire : «Suis-je ou ne suis-je pas ? ». Le poète n'hésite pas à reprendre une nouvelle fois la paraphrase de Shakespeare, en plaçant dans la bouche de son héroïne l'écho des mots d'Hamlet :

Ophélie étonnée a tâché de conclure :

«Suis-je ou ne suis-je pas? », songe-t-elle, fidèle

Au souvenir des mots d'Hamlet, seigneur volage.

(Rodenbach 1896, Aquarium mental III : 9)

La réponse ne va pas tarder :

Ses cheveux maintenant se nouent comme un feuillage

Qui jusqu'au bout de l'eau, sans fin, se ramifie.

Ophélie est trop morte, elle se liquéfie...

(Rodenbach 1896, Aquarium mental III : 9)

Cependant, quelque chose en elle semble trahir encore un semblant de vie :

Les bagues ont quitté ses mains devenant nulles;

Ses derniers pleurs à la surface font des bulles ;

Ses beaux yeux, délogés des chairs qui sont finies,

Survivent seuls, au fond, comme deux actinies.

(Rodenbach 1896, Aquarium mental III : 9)

alors que ses cheveux, eux, font penser à quelques plantes aquatiques toujours aptes à pousser :

Et ses cheveux verdis, dont la masse persiste

Dans les herbes aquatiques qui leur ressemblent,

Sont si dénaturés d'avoir trempé qu'ils semblent

Un fouillis végétal issu de cette eau triste.

(Rodenbach 1896, Aquarium mental III : 9) 
C'est un jeu de reflets continuel qui se joue ainsi entre l'âme endolorie du poète, les yeux hypnotisés de Narcisse et le geste désespéré de la jeune noyée de Shakespeare, dont les yeux ouverts sur l'eau semblent prolonger encore l'effort du mythique éphèbe. L'examen d'une âme à travers son reflet dans l'eau, la recherche acharnée de la réalité profonde des choses, sont ici à leur point culminant. Car il est clair que ce voyage aquatique dans une direction symbolique, tout droit et tout en bas, c'est encore, ça ne peut être rien d'autre sous la plume du poète symboliste que l'équivalent du retour en soi, d'une plongée dans sa vie intérieure la plus profonde et la plus obscure. Rodenbach ne cesse de le proclamer en affirmant, en des termes à peine voilés, la nécessité de compléter le regard de Narcisse par la plongée d'Ophélie :

\footnotetext{
Ah ! tout ce que le glauque aquarium enchâsse !

Ici l'eau n'est pas toute à la vie en surface,

À n'être qu'un écran docile s'imageant...

La voici, recueillie, en sa maison de verre

N'aimant plus que ce qui, dans elle, verdoie, erre

Et lui fait au dedans un Univers meilleur !
}

\section{Et le poète d'expliquer :}

Ainsi mon âme, seule, et que rien n'influence !

Elle est, comme en du verre, enclose en du silence,

Toute vouée à son spectacle intérieur,

A sa sorte de vie intime et sous-marine,

Où des rêves ont lui dans l'eau tout argentine

(Rodenbach 1896, Aquarium mental III : 7)

Le décor planté par le poète dans son « aquarium si bleuâtre et si lunaire », avec son efflorescence d'images, sa

Végétation fine, herbes, perles, lueurs ;

Et cauteleux poissons doucement remueurs

(Rodenbach 1896, Aquarium mental III : 6) 
va dans le même sens: nous sommes en présence d'un paysage d'âme. "Minéraux, végétaux et poissons symbolisent, à trois degrés différents de l'échelle de vie, le monde intérieur, qui n'est pas encore parvenu à la conscience de lui-même », écrit Paul Gorceix à propos des Vies encloses de Georges Rodenbach (Gorceix 1998 : 404). C'est bien le désir de connaitre ce "royaume glauque de l'Inconscience », comme dit le poète dans L'Ame sous-marine, désir lié à son grand projet d'instaurer la concordance primitive entre le «moi» et l'univers, qui pousse Rodenbach à poser continuellement son regard sur l'eau, ce «miroir d'éternité dont le ciel est le tain », et à le plonger dans « cette eau visionnaire », «fenêtre d'infini » (Rodenbach 1896, Aquarium mental IV : 10). Sa prédilection pour des espaces clos, dont la figuration par excellence est chez lui l'aquarium, motif très en vogue à la fin du $\mathrm{XIX}^{\mathrm{e}}$ siècle, permet de suggérer au lecteur toute l'intensité de sa quête de la vie intérieure.

Le retour massif du motif de l'eau se laisse observer encore dans le tout dernier recueil poétique de Rodenbach, Le Miroir du ciel natal, publié en 1898, l'année même de la mort de l'auteur. Là encore, l'ombre d'Ophélie semble se profiler derrière l'image de la lune, «pâle fileuse en robe blanche » qui file les jets d'eau dont on peut dire que ce sont «des cheveux qu'elle file sur un tombeau » (Rodenbach 1898, Les Jets d'eau : 112); alors qu'à un autre endroit,

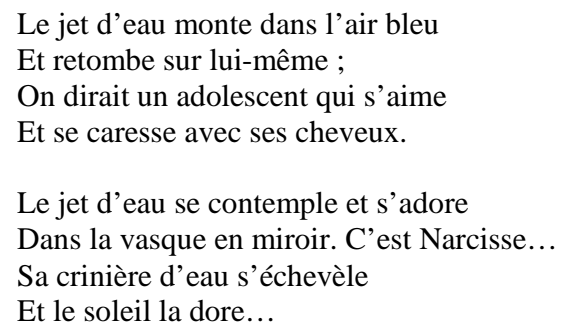

Et tout à coup, cette explication surprenante : 
Le jet d'eau rit, tel Narcisse;

$\mathrm{Car}$ - toute son eau retombant

Comme un linge qui s'éparpille -

Il semble que c'est de soi qu'il se déshabille

Et qu'il est enfin nu!

(Rodenbach 1898, Les Jets d'eau : 107-108)

N'est-ce pas l'âme du poète dans toute sa nudité, l'âme débarrassée enfin de tout ce qui lui pesait, qui se reflète ainsi dans le miroir de l'eau à l'heure suprême ? «Le jet d'eau semble à genoux » (1898: $100)$; «voilà la Lune offrant sa grande hostie » (1898: 101); « le jet d'eau songe que c'est l'heure » $(1898: 102)$; « il va baiser l'azur » (1898 : 104) ; «il est l'âme trop fière et que le ciel aimante »; «il est le doux martyr d'un idéal trop beau »; « sa robe retombe en plis agenouillés comme sur une tombe » $(1898: 106)$ - en voilà assez, sous la plume du poète, pour répondre à la question par l'affirmative.

C'est ainsi que l'âme du poète se mire dans l'eau et s'y laisse noyer. Porteuses d'un réseau d'images associant l'eau avec la mort et le mystère, les figures d'Ophélie et de Narcisse véhiculent ses angoisses, ses fascinations morbides et ses méditations sur la vie intérieure. Alors que l'eau d'Ophélie est envisagée comme une eau dormante qui appelle vers les profondeurs, comme le constate dans son article sur le mythe d'Ophélie fin de siècle Anne Cousseau (Cousseau 2001 : 88), celle de Narcisse apparaît, en conformité avec la version ovidienne, comme surface réfléchissante. Les deux se croisent et se complètent chez Rodenbach pour donner l'image non plus du cœur, à la manière des romantiques, mais de l'âme; en d'autres termes, pour accompagner une recherche de la spiritualité propre aux écrivains symbolistes : celle qui consiste à doter le rêve d'un pouvoir de traverser les apparences du monde réel.

Bibliographie

Rodenbach G. (1886) : La Jeunesse blanche. Paris : Alphonse Lemerre.

Rodenbach G. (1891) : Le Règne du silence. Paris : Bibliothèque-Charpentier.

Rodenbach G. (1896) : Les Vies encloses. Paris : G. Charpentier et E. Fasquelle.

Rodenbach G. (1898) : Le Miroir du ciel natal. Paris : Fasquelle. 
Bachelard G. (1942) : L'eau et les rêves. Paris : José Corti.

Cousseau A. (2001): Ophélie: histoire d'un mythe fin de siècle. Revue d'histoire littéraire de la France, 101(1), pp. 81-104, 105-122.

Eymard J. (1977) : Ophélie ou le narcissisme au féminin. Étude sur le thème du miroir dans la poésie féminine (XIX ${ }^{e}-X X^{e}$ siècles). Paris : Minard.

Gorceix P. dir. (1998) : Fin de siècle et Symbolisme en Belgique. Euvres poétiques. Bruxelles : Éditions Complexe.

Malinowski W. M. (2005): 'L'ophélisation d'une ville entière'. Sur le rôle de l'élément aquatique dans 'Bruges-la-Morte' de Georges Rodenbach. In : M. P. Mrozowicki (dir.), In aqua scribis. Le thème de l'eau dans la littérature. Gdańsk : Wydawnictwo Uniwersytetu Gdańskiego, pp. 137-144.

Sobierajska A. (2014) : Le mythe d'Ophélie dans la littérature belge d'expression française à l'époque du symbolisme. Thèse de doctorat préparée sous la direction de Wiesław Mateusz Malinowski et soutenue à l'Université Adam Mickiewicz de Poznań. 